\title{
Inhalt der 2. Lieferung
}

Erster Abschnitt: Vorschriften für sämtliche Vszweige

Zweiter Titel: Anzeigepflicht. Gefahrerhöhung . . . . . . . . . . . . 314-450

$\S 16$ Vorvertragliche Anzeigepflicht. Nichtanzeige . . . . . . . . 314-335

$\S 17$ Falschanzeige . . . . . . . . . . . . . . . . . . 335-337

$\$ 18$ Fragebogen . . . . . . . . . . . . . . . . . . 337-339

$\$ 19$ Mitwirkung Drittbeteiligter . . . . . . . . . . . . 339-343

$\S 20$ Rücktritt: Frist, Erklärung, Wirkungen . . . . . . . . . . . 343-349

$\S 21$ Rücktritt: Einschränkung der Wirkungen . . . . . . . . . 349-354

$\$ 22$ Anfechtung, Nichtigkeit des Vertrages . . . . . . . . . . . 355-373

$\$ 23$ Gefahrerhöhung. Obliegenheiten bei subjektiver Gefahrerhöhung 374-389

$\S 24$ Kündigung bei subjektiver Gefahrerhöhung . . . . . . . . . 389-392

$\S 25$ Leistungsfreiheit bei subjektiver Gefahrerhöhung . . . . . . . 392- 395

$\S 26$ Subjektive Gefahrerhöhung ohne Rechtsïolgen . . . . . . . . 395-397

$\S 27$ Rechtsfolgen bei objektiver Gefahrerhöhung . . . . . . . . . 397-399

$\$ 28$ Speziell: Leistungsfreiheit bei objektiver Gefahrerhöhung . . . 399-401

\$29 Gefahrerhöhung ohne Rechtsfolgen . . . . . . . . . . . . . 401-404

$\$ 29$ a Gefahrerhöhung nach Antragstellung . . . . . . . . . . . . 404-406

$\S 30$ Teilrücktritt, Teilkündigung, teilweise Leistungsfreiheit, Teil-

anfechtung . . . . . . . . . . . . . . . .406-411

$\$ 31$ (gestrichen) . . . . . . . . . . . . . . . . . . . . . 411

$\S 32$ Vorbeugende Obliegenheiten . . . . . . . . . . . . . . . . 412-423

$\S 33$ Obliegenheit zur Anzeige des Versicherungsfalles . . . . . . . . 424-430

$\S 34$ Auskunfts- und Belegpflicht. Täuschung bei Schadens-

ermittlung . . . . . . . . . . . . . . . . . 431-446

\$34a Unabdingbarkeit, Schriftform . . . . . . . . . . . . . . . 446-450

Dritter Titel: Prämie. . . . . . . . . . . . . . . . . . 450-535

$\S 35$ Vorbemerkungen. Prämienzahlung. . . . . . . . . . . .450-476

$\S 35$ a Befriedigungsrecht, Pfandrechtserweiterung . . . . . . . . . 476-479

$\S 35 \mathrm{~b}$ Abzugsrecht . . . . . . . . . . . . . . . . . . . . . . . 479-480

$\S 36$ Leistungsort, Leistungszeit . . . . . . . . . . . . . . . . . 481-486

$\$ 37$ Prämienabholung . . . . . . . . . . . . . . . . . . . . . . 486-488

$\S 38$ Prämienverzug bei Erstprämie . . . . . . . . . . . . . . . 489-498

$\S 39$ Prämienverzug bei Folgeprämie . . . . . . . . . . . . . . 498-513

$\S 40$ Frage der Unteilbarkeit der Prämie . . . . . . . . . . . . . 513-519

$\$ 41$ Prämienunzulänglichkeit, Prämienerhöhung . . . . . . . . . 519-530

$\$ 41$ a Prämienherabsetzung. . . . . . . . . . . . . . . . . . 530-534

$\S 42$ Unabdingbarkeit . . . . . . . . . . . . . . . . 534-535

Abkürzungen

Die Abkürzungen für die gebräuchlichsten Allgemeinen Versicherungsbedingungen (AVB) sind eingeführt in der Einleitung Anm. 20 (Lieferung 1).

Das wichtigste Schrifttum ist mit der benutzten Zitierweise angeführt in der Einleitung Anm. 39 (Lieferung 1). - Ist ein Werk mit dem Zusatz a. a. O. zitiert, so ist der genaue Fundort aus den Schriftumsangaben des betreffenden Absehnitts zu entnehmen.

Ferner bedeuten: $\mathrm{V}=$ Versicherung, Ver $=$ Versicherer, Vmer $=$ Versicherungsnehmer, Vter $=$ Versicherter.

Ein ausführliches Abkürzungsverzeichnis wird der letzten Lieferung beigefügt sein. 


\section{Zwingende Vorschriften.}

\section{Gliederung :}

Entstehung Anm. 1

Schrifttum Anm. 2

I. Arten zwingender Vorschriften Anm.3
II. Relativ zwingende Vorschriften Anm. 4

III. Absolut zwingende Vorschriften Anm. 5

IV. Nicht zwingende Vorschriften Anm. 6

[1] Entstehung: $\S 15$ a ist durch die VO vom 9. XII. 1939 eingefügt worden in dem Bestreben, jeweils am Ende eines Gesetzestitels die zwingenden Normen übersichtlich zusammenzustellen. - Begr. III S. 7.

[2] Sehrifttum: Vgl. Einl. Anm. 40.

\section{[3] I. Arten zwingender Vorschriften.}

Man muB relativ und absolut zwingende Normen unterscheiden (Einl. Anm. 45-50). Der $\$ 15$ a beschränkt sich auf die Zusammenstellung relativ zwingender Vorschriften, aber gerade der Erste Titel enthält auch mehrere absolut zwingende Bestimmungen.

Alle Beschränkungen der Vertragsfreiheit gelten nicht nur für endgültige Vsverträge, sondern auch für vorläufige Deckungszusagen (Anm.94 zu §1).

\section{[4] I. Relativ zwingende Vorschriften.}

Der Ver kann sich nicht auf eine Vereinbarung berufen, durch welche von gewissen, in $\$ 15$ a genannten Vorschriften zum Nachteile des Vmers abgewichen wird. Die Vereinbarung ist aber durchaus juristisch existent, sie ist nicht nichtig. Das zeigt sich darin, daß der Vmer, ein Vter, ein Bezugsberechtigter, überhaupt jede Person außer dem Ver (oder seinem Rechtsnachfolger) sich auf die Vereinbarung berufen kann. Das hat besonders dann praktische Bedeutung, falls eine Vereinbarung den Vmer usw. im Vergleich zur gesetzlichen Rechtslage teils schlechter, teils besser stellt. Hinsichtlich der Besserstellungen wird der Vmer sich regelmäßig auf das Vereinbarte stützen. Näheres Einl. Anm. 49.

Den Abweichungen zum Nachteile des Vmers stehen regelmäBig solche zum Nachteile des Vten, Bezugsberechtigten, Zessionars usw. gleich. Auch eine Umgehung stellt sich als Abweichung dar.

Im Einzelnen wird auf die Ausführungen zu den in $\$ 15$ a genannten Normen verwiesen, nämlich zu

§ 3 III auf Anm. 38 zu $\S 3$,

$\S 5$ I-III auf Anm. 26 zu $\S 5$,

$\S 6$ I-III auf Anm. $110 \mathrm{zu} \S 6$,

$\S 8$ II auf Anm. 17 zu $\S 8$,

$\S 11$ II auf Anm. 31 zu $\$ 11$,

$\S 12$ aul Anm. 54-57 zu $\$ 12$,

$\S 14$ auf Anm. 6, 20, 24 zu $\S 14$.

Alle Bestimmungen sind nur relativ zwingend im Bereich der Beschränkungen der Vertragsfreiheit; dazu Einl. Anm. 41.

\section{[5] III. Absolut xwingende Vorschriften.}

Die absolut zwingenden Bestimmungen sind durchweg schwieriger zu ermitteln als die relativ zwingenden. Sie können weder zum Nachteil noch zum Vorteil des Vmers oder Vers wegbedungen werden. Sie setzen sich auch im Bereich der Beschränkungen der Vertragsfreiheit durch. Näheres Einl. Anm. 42.

Das Gesetz selbst läßt den absolut unabdingbaren Charakter folgender Vorschriften erkennen:

§ 5 IV, dazu Anm. 23, 26 zu §5,

§ 6 IV, dazu Anm. 20, 110 zu $\$ 6$,

\$ 8 I, dazu Anm. 5-7 zu §8,

$\$ 11$ IV, dazu Anm. 27 zu \$11.

Im Wege der Auslegung muß man zu dem Ergebnis kommen, daß auch folgende 
Anm. 6

Vorschritten absolut zwingende Normen enthalten:

\$1 I 1 (vsrechtliches Bereicherungsverbot), vgl. Einl. Anm. 42, Anm. 26 zu §1, \$4 I (Vsschein kein Inhaberwertpapier), vgl. Anm. $32 \mathrm{zu} \S 3$, Anm. $10 \mathrm{zu} \S 4$,

\$13 (Vertragsbeendigung durch Verkonkurs), vgl. Anm. 21 zu §13,

$\S 15$ (Abtretungsverbot bei V unpfändbarer Sachen), vgl. Anm. 29 zu $\S 15$.

In gewissem Sinne sind auch zu nennen:

\$1II (Gleichstellung von Prämien und Beiträgen im Rahmen zwingender Bestimmungen z. B. $\S \S 38,39$, vgl. Anm. 36, 39 zu §1),

$\S 9$ (Höchstdauer der Vsperiode im Rahmen zwingender Bestimmungen,vgl. Anm. ? zu \$9).

[6] IV. Nicht zwingende Vorsehriften.

Abdingbar sind z. B. § 2 (Anm. 43 zu §2), § 3 I, II, IV (Anm. 14, 35 zu § 3), §4 II, § 7 (Anm. 5 zu § 7), §9 (Anm. 4, 7 zu $\S 9$, vgl. aber auch oben Anm. 5), § 10 (Anm. 21 zu §10), §11 I, III (Anm. 10-12 zu §11).

Als abdingbar kann auch $\$ 1$ I 2 bezeichnet werden, da eine Personenv nicht nur als Summenv, sondern auch als Schadensv betrieben werden kann (Anm. 26 zu §1).

Zweiter Titel.

Anzeigepflicht. Gefahrerhöhung.

\section{$\$ 16$}

(1) Der Versicherungsnehmer hat bei der Schliebung des Vertrags alle ihm bekannten Umstände, die für die Ǔbernahme der Gefahr erheblich sind, dem Versicherer anzuzeigen. Erheblich sind die Gefahrumstände, die geeignet sind, auf den Entschluß des Versicherers, den Vertrag überhaupt oder zu dem vereinbarten Inhalt abzuschließen, einen Einfluß auszniiben. Ein Umstand, nach welchem der Versicherer ausdriicklich und schriftlich gefragt hat, gilt im Zweifel als erheblich.

[2] Ist dieser Vorschrift zuwider die Anzeige eines erheblichen Umstandes unterblieben, so kann der Versicherer von dem Vertrage zurïektreten. Das Gleiche gilt, wenn die Anzeige eines erheblichen Umstandes deshalb unterblieben ist, weil sich der Versicherungsnehmer der Kenntnis des Umstandes arglistig entzogen hat.

[3] Der Rücktritt ist ausgeschlossen, wenn der Versicherer den nicht angezeigten Umstand kannte oder wenn die Anzeige ohne Verschulden des Versicherungsnehmers unterblieben ist.

Vorvertragliche Anzeigepflicht.

Gliederung:

Entstehung Anm. 1

Schrifttum Anm. 2

I. Stoffgliederung Anm. 3

II. Rechtsnatur Anm. 4-5

III. Tatbestand Anm. 6-38

1. Anzeigepflichtiger Anm. 6

2. Anzeigeempfänger Anm. 7

3. Zeit Anm. 8-11

a) Allgemeines Anm. 8

b) Sonderfälle Anm. 9-10

c) Umgehungen Anm. 11

4. Ort Anm. 12

5. Form Anm. 13

\section{Nichtanzeige.}

6. Inhalt Anm. 14-37

a) Gefahrumstände Anm. 15-24

aa) Kausale und indizierende Umstände Anm. 16

bb) Vsgefahr- und Vertragsgefahrumstände Anm. 17

cc) Positive und negative Umstände Anm. 18

dd) Objektive und subjektive Umstände Anm. 19

ee) Verschuldete und unverschuldete Umstände Anm. 20 